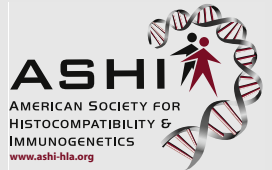

journal homepage: www.elsevier.com/locate/humimm

\title{
Myeloid blood dendritic cells and monocyte-derived dendritic cells differ in their endocytosing capability
}

\author{
Linda I.M. Andersson, Emina Cirkic, Peter Hellman, Håkan Eriksson* \\ Department of Biomedical Laboratory Science, Faculty of Health and Society, Malmö University, S-205 06 Malmö, Sweden
}

\section{A R T I C L E I N F O}

\section{Article history:}

Received 28 December 2011

Accepted 9 August 2012

Available online $\mathrm{xxxx}$

\begin{abstract}
A B S T R A C T
Human dendritic cells (DCs) constitute a heterogeneous population of antigen-presenting cells characterized by a unique capacity to stimulate naïve T cells. The functions of DCs depend on the particular subset and in this study we compare two types of myeloid DCs: freshly isolated blood mDCs and in vitro generated monocyte-derived DCs (MoDCs), in their ability to accomplish endocytosis.

In our hands, these two DC subtypes showed similarities in the expression of surface markers, but displayed clear differences in endocytic capacity. Freshly isolated blood mDCs showed a high propensity to capture and endocytose particles compared to in vitro generated MoDCs. The blood mDCs also showed a clear receptor-enhanced endocytosis when zeolite particles were co-adsorbed with IgG. On the other hand, the MoDCs differed remarkably compared to blood mDCs in the capture of ovalbumin and immune complexes. Interestingly, the MoDCs showed low endocytosis of IgG-coated particles but an efficient capture of immune complexes. The MoDCs also showed a high capacity to capture ovalbumin although with a relatively low degree of internalization. These data indicate distinct differences in the early process of endocytosis featured by mDCs and MoDCs, which is important to consider when choosing DC populations for future functional or clinical applications.
\end{abstract}

(c) 2012 American Society for Histocompatibility and Immunogenetics. Published by Elsevier Inc. All rights reserved.

\section{Introduction}

Dendritic cells (DCs) constitute a central part of our immune system. DCs are rare bone marrow-derived cells, involved in antigen capture, processing and presentation [1]. The DCs ability to endocytose, process and present antigens is highly dependent upon their functional state. Immature DCs possess high endocytic capacity, and are specialized to capture and process antigens but are less potent to initiate T-cell activation [2]. A variety of soluble factors and pathogen signals are known to activate DCs and the mature state is accompanied by attenuated endocytosis and facility to trigger a T-cell immune response. In vitro maturation is reflected by an increased capacity to extend dendritic projections, increased antigen presentation and up-regulation of MHC class II and co-stimulatory molecules such as CD40, CD80, CD83 and CD86 [3,4].

DCs can be divided into a variety of subtypes with different localizations and specialized functions regarding immune responses. Using human sources, different DCs can be generated

\footnotetext{
* Corresponding author.

E-mail address: hakan.eriksson@mah.se (H. Eriksson).
}

in vitro from; $\mathrm{CD} 34^{+}$precursor cells from bone marrow, cord blood, peripheral blood, or from blood monocytes. Due to the scarcity of blood myeloid DCs (mDCs), the majority of human ex vivo DC studies have been performed on DC-like cells (MoDCs) that have been differentiated from peripheral blood monocytes by granulocytemacrophage colony stimulating factor (GM-CSF) and interleukin4 (IL-4) [5].

In vivo, DCs perform the role of sentinel cells, continually searching for signs of infection by internalizing fluid samples and cellular debris by different endocytosing mechanisms; particulate antigens and microbes are taken up by phagocytosis, while extracellular fluids, soluble antigens and small particles are captured by macropinocytosis, receptor-mediated endocytosis or pinocytosis [6-8]. Endocytosis is essential in the regulation of signal transduction, immune surveillance, antigen presentation, crosspresentation and maintaining cellular homeostasis [9].

In this report, we describe and compare the antigen capturing capacity of freshly isolated mDCs and in vitro generated MoDCs with respect to different endocytosing mechanisms using dealuminated zeolite particles [15,20,21], immune complex and soluble molecules. Freshly isolated mDCs and MoDCs were phenotypically similar, and were also alike in the expression of co-stimulatory 
markers, but differed in the capacity to endocytose particles, immune complexes and soluble molecules.

\section{Materials and methods}

\subsection{Reagents and zeolites}

Alexa Fluor ${ }^{\circledR} 488$ Ovalbumin conjugate (Alexa-OA) and $\mathrm{DQ}^{\mathrm{TM}}$ Ovalbumin (DQ-OA) were obtained from Molecular probes, Inc. Eugene, OR, USA and the IgG fraction of an Anti-ovalbumin (Hen egg white) (Rabbit) serum came from Rockland Immunochemicals, Gilbertsville, PA, USA). Human immunoglobulin was obtained from Octapharma, Stockholm, Sweden and cytokines, GM-CSF and IL-4, were purchased from PeproTech EC, London, UK. All other reagents were of analytical grade.

Surface phenotype analysis was performed using monoclonal antibodies against CD11c, CD32, CD40, CD64, CD80, CD86 and MHC II from Becton Dickinson, San Jose, CA, USA and CD14 from Serotec, Oxford, UK. Dealuminated zeolite, USY (Zeolite Y, Si/Al ratio: 240) was manufactured by Tosoh cooperation, Japan. Zeolites used in cell experiments were sterilized by heating for $3 \mathrm{~h}$ at $300{ }^{\circ} \mathrm{C}$ before use.

\subsection{Cell preparation}

MACS technology based on magnetically labeling cells and retaining cells on a column was used to isolate mDCs (CD1c (BDCA-1) ${ }^{+}$dendritic cell isolation kit) and monocytes (Monocyte isolation kit II) (Miltenyi Biotec, Bergisch Gladbach, Germany).

Briefly, peripheral blood mononuclear cells (PBMC) were obtained from buffy coat from healthy donors by density-gradient centrifugation on Ficoll-Paque ${ }^{\mathrm{TM}}$ (Amersham Pharmacia Biotech $\mathrm{AB}$, Uppsala, Sweden) with added fragmin, 20 units/ml, (Pfizer, Täby, Sweden).

Immature blood mDCs were isolated by a two-step procedure; in the first step magnetically labeled B cells (CD19) were depleted and in the second step, mDCs were isolated using microbeads and antibodies against BDCA-1. To achieve the highest purity of the $\mathrm{mDC}$ population, the positively selected cells were separated on a second MACS column as a standard procedure. The cells were resuspended in RPMI 1640 medium supplemented with $10 \%$ human serum "off the clot", Type AB, (PAA laboratories, Linz, Austria), and $100 \mu \mathrm{g} / \mathrm{ml}$ of gentamicin (PAA laboratories, Linz, Austria). This medium will be referred to as R10. All cells were cultured at $37{ }^{\circ} \mathrm{C}$ in a humidified atmosphere containing $5 \% \mathrm{CO}_{2}$.

Untouched $\mathrm{CD}_{14}{ }^{+}$monocytes were isolated by indirect magnetic labeling of non-monocytes with a cocktail of biotin-conjugated antibodies against CD3, CD7, CD16, CD19, CD56, CD123, and CD235a followed by the addition of anti-Biotin MicroBeads. Non CD14 $4^{+}$monocytes were depleted on a MACS column and cells were re-suspended in R10 medium. To generate immature MoDC, human peripheral blood monocytes were further cultured for 7 days in RPMI 1640 medium containing 10\% FCS (PAA laboratories, Linz, Austria), 2\% human serum, $150 \mathrm{ng} / \mathrm{ml} \mathrm{rGM-CSF}$ and $50 \mathrm{ng} / \mathrm{ml} \mathrm{IL-4}$ (PeproTech EC, London, UK). Half of the medium volume was exchanged every 2-3 days [10].

\subsection{Preparation of zeolites}

Preparation of zeolites were done as earlier described [11] and briefly dealuminated zeolite Y, $50 \mathrm{mg}$, was washed with $20 \mathrm{mM}$ Tris-buffer $\mathrm{pH} 7.4$ (Tris-buffer) and collected by centrifugation for $5 \mathrm{~min}$ at $13,000 \times \mathrm{g}$. Zeolites were re-suspended in $1 \mathrm{ml}$ Trisbuffer, divided into $250 \mu \mathrm{l}$ aliquots and stored at $-20^{\circ} \mathrm{C}$. A frozen aliquot of zeolite, $250 \mu \mathrm{l}$, was suspended in $1.25 \mathrm{ml}$ Tris-buffer and left at room temperature to sediment. After one hour of sedimentation, $1 \mathrm{ml}$ of the supernatant was collected $(1 \times g$ supernatant) and the amount of zeolite particles in the supernatant was quantified using light scattering $(800 \mathrm{~nm})$ and the following formula:

$$
\frac{\text { Abs800nm }}{0.63}-0.10=\mathrm{mg} \text { zeolite } \mathrm{Y} / \mathrm{ml}
$$

\subsection{Coating zeolites}

Coating of zeolites were done as earlier described [15,20,21] and briefly volumes of $1 \times g$ supernatants corresponding to $1 \mathrm{mg}$ zeolite particles were centrifuged for $5 \mathrm{~min}$ at $13,000 \times \mathrm{g}$ and the collected zeolites were suspended and incubated in $0.5 \mathrm{ml}$ of Tris-buffer containing Alexa-OA or DQ-OA on a rocking table for $1 \mathrm{~h}$ using a final concentrations of $0.2 \mathrm{mg} / \mathrm{ml}$ Alexa-OA or DQ-OA. The zeolites were collected and washed twice with $1 \mathrm{ml}$ PBS.

Zeolites coated with human IgG were first pre-coated with Alexa-OA as described above and after being washed, $1 \mathrm{mg}$ of the pre-coated zeolites were incubated on a rocking table for $1 \mathrm{~h}$ with $0.5 \mathrm{mg} / \mathrm{ml}$ human IgG. The IgG-coated zeolites were collected and washed twice with $1 \mathrm{ml}$ PBS. As a control, $1 \mathrm{mg}$ zeolite from the $1 \times g$ supernatant was incubated with Tris-buffer on a rocking table for $1 \mathrm{~h}$ and washed as described above.

\subsection{Incubation with zeolite particles}

Incubation of cells and zeolites were done as earlier described $[15,20,21]$ and briefly cells, $1 \times 10^{6}$ cells/ml in R10 medium, were incubated with ligand-coated zeolites in a final concentration of $40 \mu \mathrm{g} / 10^{6}$ cells for $4 \mathrm{~h}$ at $37^{\circ} \mathrm{C}$. As a control, cells were incubated for $4 \mathrm{~h}$ at $37^{\circ} \mathrm{C}$ with a control containing un-coated zeolite suspended in R10 as described above. After incubation, the cells were washed with $1 \mathrm{ml}$ PBS containing $0.1 \%(\mathrm{w} / \mathrm{v})$ BSA (PBS-BSA), resuspended in $500 \mu \mathrm{l}$ PBS-BSA and analysed by FACScan using standard settings and CELLQuest version 3.3 (Becton Dickinson, San Jose, CA, USA).

\subsection{Expression of surface markers}

Cells were washed with $1 \mathrm{ml}$ PBS containing 0.1\% PBS-BSA, resuspended in $100 \mu \mathrm{l}$ PBS containing $0.1 \%(\mathrm{w} / \mathrm{v}) \mathrm{BSA}$ and $0.1 \%(\mathrm{w} / \mathrm{v})$ human IgG (PBS-BSA-hIgG) and stained with fluorescein isothiocyanate (FITC), phycoerythrin (PE) or Cy5-PE conjugated monoclonal antibodies against CD11c, CD14, CD32, CD64, CD40, CD80, CD83, CD86, MHC II, CD206 and isotype controls on ice for 30 min. Cells were washed and analysed by FACScan using standard settings and CELLQuest version 3.3.

\subsection{Immune complexes}

Antigen-antibody complexes (IC) were obtained by incubating the IgG fraction of an anti-OA $(477 \mu \mathrm{g} / \mathrm{ml})$ serum with ovalbumin conjugated with Alexa Fluor $488(12 \mu \mathrm{g} / \mathrm{ml})$ for $1 \mathrm{~h}$ at $37^{\circ} \mathrm{C}$. Cells, $5 \times 10^{5}$ cells $/ \mathrm{ml}$, were then incubated with IC at a final concentration corresponding to $0.25 \mu \mathrm{g} / \mathrm{ml}$ Alexa-OA for $1 \mathrm{~h}$ at $37^{\circ} \mathrm{C}$. After incubation, the cells were washed with $1 \mathrm{ml}$ PBS containing $2 \%$ (w/w) human serum and re-suspended in $500 \mu \mathrm{l}$ PBS-BSA and analysed by FACScan using standard settings.

\subsection{Capture of Alexa-OA and $D Q-O A$}

Cells, $5 \times 10^{5}$ cells $/ \mathrm{ml}$ in R10 medium, were incubated with Alexa-OA or DQ-OA at a final concentration of $1 \mu \mathrm{g} / \mathrm{ml}$ for $1 \mathrm{~h}$ 


\section{A. MoDCs}

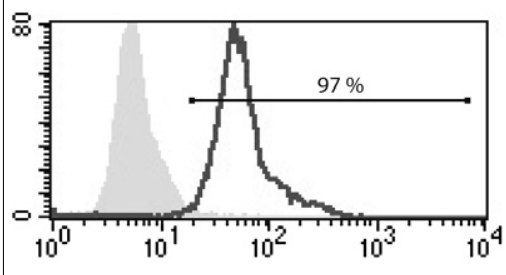

CD11c

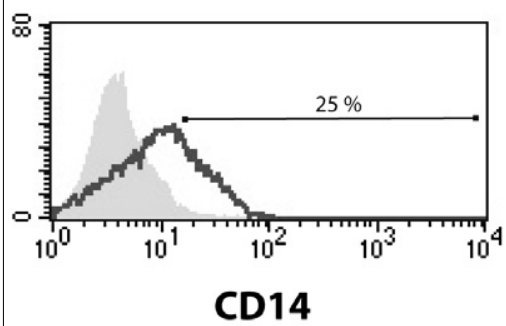

\section{B. Blood mDCs}
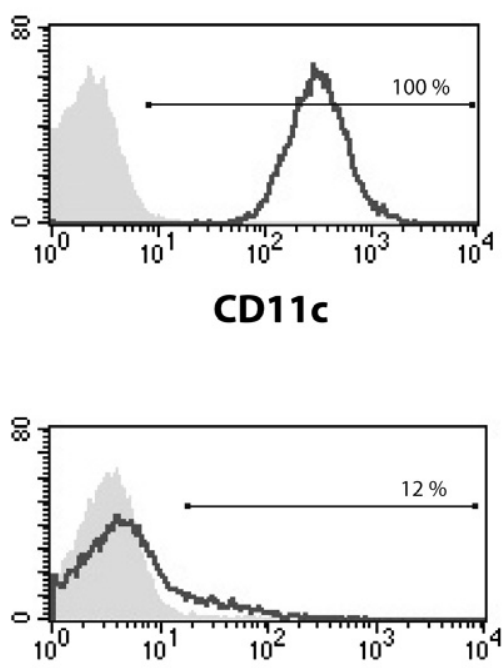

CD14

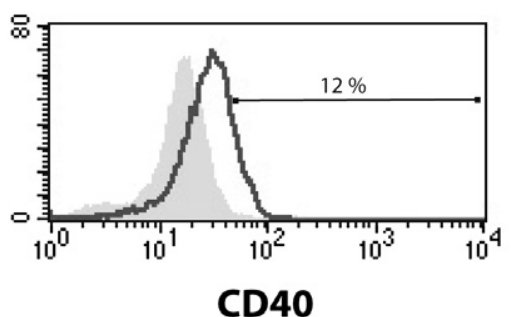

CD40

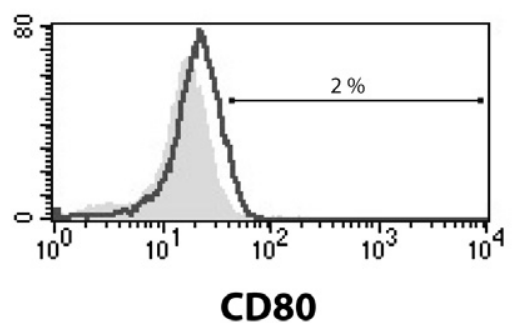

CD80
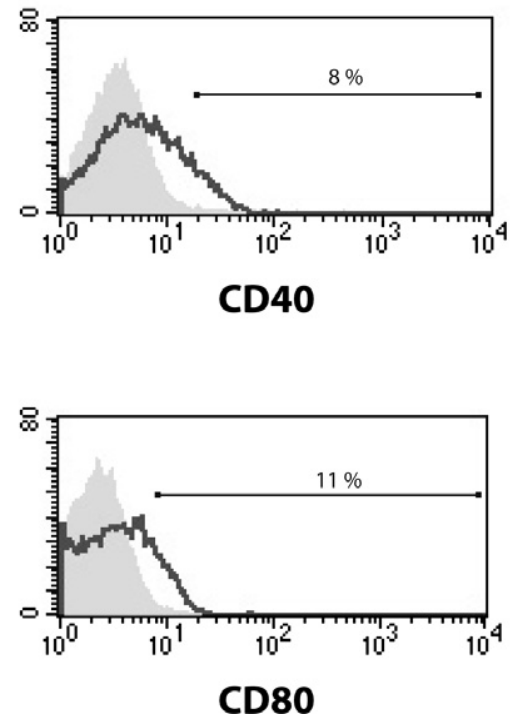
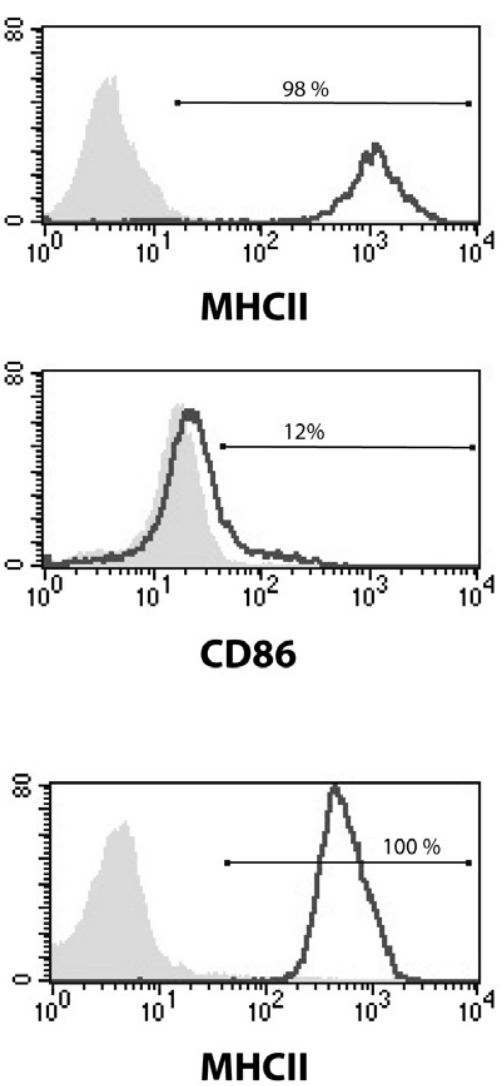

CD86

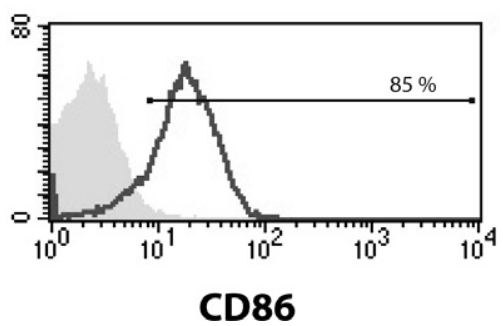

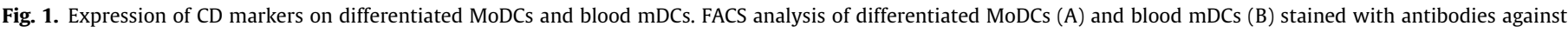

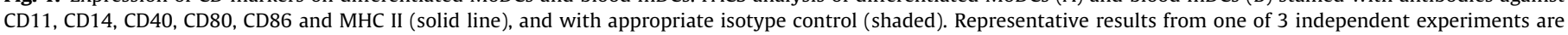
shown. Bar indicate \% positive cells with one \% or less positive cells in the isotype control.

at 37 or $4{ }^{\circ} \mathrm{C}$. After incubation, the cells were washed twice with $1 \mathrm{ml}$ PBS containing $2 \%$ human serum, re-suspended in

\section{Table 1}

Phenotype of human peripheral mDCs and MoDCs Mean fluorescence intensity (MFI) from three independent preparations of MoDCs and mDCs after staining with antibodies against surface markers.

\begin{tabular}{|c|c|c|c|c|}
\hline \multirow{2}{*}{$\begin{array}{l}\text { Surface } \\
\text { marker }\end{array}$} & \multicolumn{2}{|l|}{ MoDCs } & \multicolumn{2}{|l|}{$\mathrm{mDCs}$} \\
\hline & $\begin{array}{l}\text { Specific mab } \\
\text { MFI } \pm \mathrm{sd}\end{array}$ & $\begin{array}{l}\text { Isotype } \\
\text { control } \\
\text { MFI } \pm \text { sd }\end{array}$ & $\begin{array}{l}\text { Specific mab } \\
\text { MFI } \pm \text { sd }\end{array}$ & $\begin{array}{l}\text { Isotype } \\
\text { control } \\
\text { MFI } \pm \text { sd }\end{array}$ \\
\hline CD11c & $60 \pm 26$ & $5 \pm 1$ & $301 \pm 78$ & $4 \pm 3$ \\
\hline CD14 & $8 \pm 3$ & $4 \pm 1$ & $6 \pm 3$ & $5 \pm 2$ \\
\hline CD40 & $20 \pm 5$ & $7 \pm 6$ & $4 \pm 2$ & $3 \pm 2$ \\
\hline CD80 & $13 \pm 5$ & $10 \pm 5$ & $4 \pm 2$ & $3 \pm 2$ \\
\hline CD86 & $25 \pm 16$ & $10 \pm 5$ & $22 \pm 10$ & $2 \pm 1$ \\
\hline MHC class II & $620 \pm 319$ & $4 \pm 1$ & $573 \pm 75$ & $4 \pm 2$ \\
\hline
\end{tabular}

$500 \mu \mathrm{l}$ PBS-BSA and analysed by FACScan using standard settings.

\section{Results}

The aim of this paper was to investigate whether or not CD14 monocytes differentiated into immature MoDCs are an applicable cell model of peripheral blood dendritic cells with respect to their endosomal processes and pathways. The differentiation of CD14 monocytes into immature MoDCs for 7 days with GM-CSF and IL4 showed in agreement with other studies [12-14], a high expression of the surface markers $\mathrm{CD} 11 \mathrm{c}^{+}$and MHC class II together with a low expression of CD14, CD80, CD86 and CD40 (Fig. 1A). Freshly isolated blood mDCs showed a similar expression of surface markers (Fig. 1B) as MoDCs and Table 1 shows the average mean fluorescence intensity (MFI) from three independent preparations of MoDCs and mDCs after staining with antibodies against the surface markers CD11c, CD14, CD40, CD80, CD86 and MHC class II. 


\subsection{Capture of zeolite particles}

The endocytosing/phagocytosing capacity of freshly isolated blood mDCs, monocytes and differentiated immature MoDC was determined by a $4-\mathrm{h}$ incubation at $37{ }^{\circ} \mathrm{C}$ with zeolite particles. To facilitate tracing and identification of the zeolite particles, the zeolite was surface coated with $\mathrm{OA}$ conjugated with the $\mathrm{pH}$-insensitive FITC-like fluorescent dye Alexa Fluor 488 (Alexa-OA). Further coadsorption of human IgG onto pre-coated Alexa-OA particles was done to study receptor-enhanced endocytosis of particles. As described earlier, no effect on the fluorescence intensity of the particles was observed due to co-adsorption of human IgG [15].

Flow cytometry was used to determine the capture of zeolite particles and cells incubated with non-coated zeolite particles were used to determine the background fluorescence of the cells. Representative experiments are shown in Fig. 2 with one single population of immature MoDC having a low uptake of Alexa-OA coated zeolites (Fig. 2A) in comparison with blood mDCs that consist of one population with high uptake of particles and one with a much lower uptake of zeolite particles (Fig. 2B). Compared to
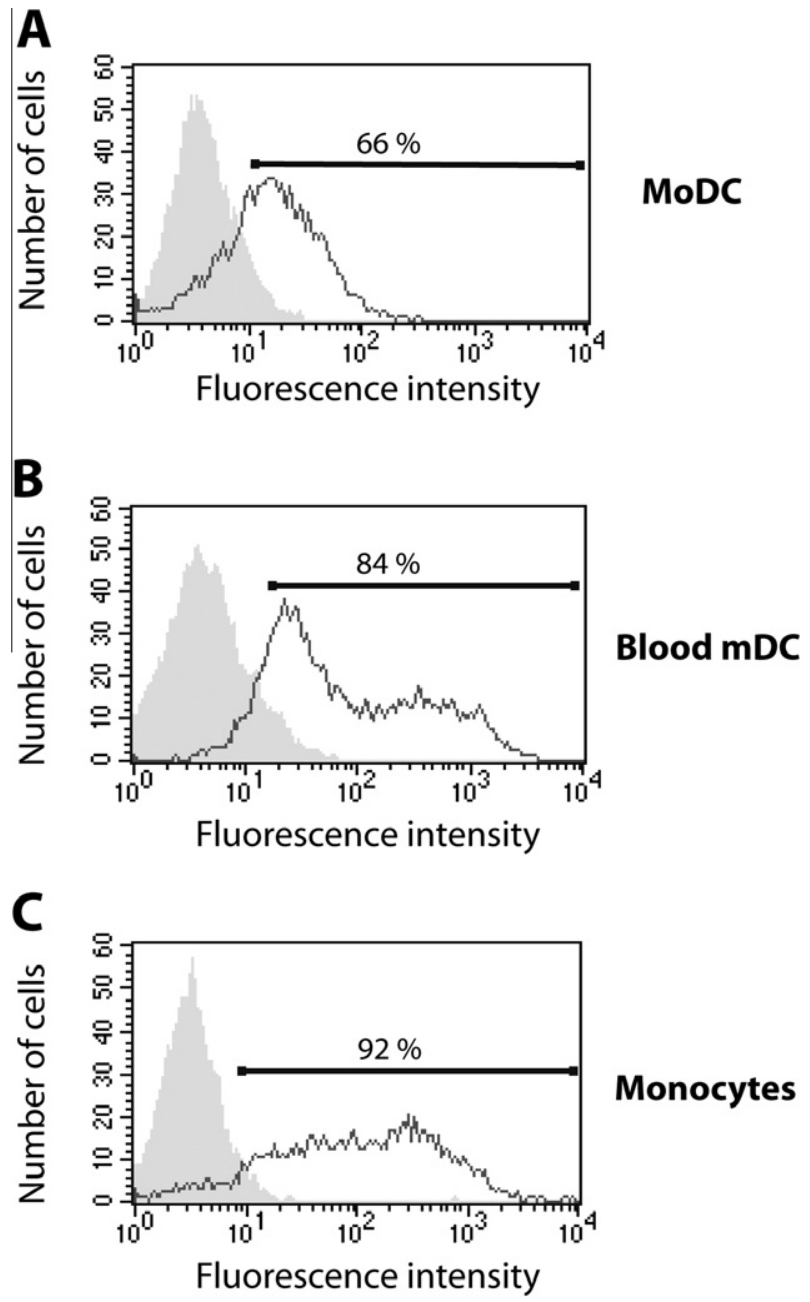

Fig. 2. Endocytosis of zeolite particles by differentiated MoDCs and freshly isolated blood mDCs and monocytes. Overlay figures showing endocytosis of zeolite particles coated with Alexa-OA (solid line) or control, un-coated zeolite, (shaded) after incubation for $4 \mathrm{~h}$ at $37^{\circ} \mathrm{C}$. (A) MoDCs, (B) peripheral mDCs and (C) monocytes. Representative results from one of 3 independent experiments are shown. Bar indicate \% positive cells with five \% or less positive cells in the un-coated zeolite control. MFI after incubation between particles and three independent cell preparations; MoDCs: Alexa-OA zeolite $9 \pm 5$ and un-coated zeolite $4 \pm 1$, mDCs: Alexa-OA zeolite $124 \pm 47$ and un-coated zeolite $8 \pm 6$ and monocytes: Alexa-OA zeolite $363 \pm 239$ and un-coated zeolite $8 \pm 5$. immature MoDC and blood mDCs, the monocyte population showed a more efficient capture of particles, consistent with being specialized phagocytes (Fig. 2C).

Peripheral monocytes, MoDCs and mDCs all express Fc-receptors (Fig. 3) and the cell populations showed a high expression of the Fc $\gamma$-receptor CD32, although a difference was observed regarding the Fc $\gamma$-receptor CD64. The MoDC population showed no detectable expression of CD64 whereas both monocytes and mDCs expressed CD64. Table 2 shows the average MFI from three independent preparations of monocytes, MoDCs and mDCs stained with antibodies against the Fc-receptors CD32 and CD64.

Co-adsorption of IgG onto Alexa-OA labeled zeolite particles resulted in higher capture and endocytosis of the particles (Fig. 4). This was in agreement with earlier results showing co-adsorption of IgG to enhance the capture and endocytosis of particles through interactions with Fc-receptors expressed by the cells [15]). A strong receptor enhanced capture due to IgG coating was in particular seen from monocytes and mDCs. MoDC showed a much lower capture of IgG coated particles (Fig. 4).

\subsection{Capture and internalization of immune complex (IC)}

To investigate whether freshly isolated blood mDCs and differentiated immature MoDCs differed in IC capture and endocytosis, cells were incubated for $1 \mathrm{~h}$ at $37^{\circ} \mathrm{C}$ with IC prepared from purified anti-OA IgG and Alexa-OA. All cell preparations showed capture of IC (Fig. 5), however, MoDCs showed a much higher degree of capture compared to $\mathrm{mDCs}$ and showed a capture of IC resembling the capture by monocytes.

\subsection{C-type lectin receptor}

Freshly isolated blood mDCs and immature MoDCs differed in their particle endocytosis and receptor-mediated endocytosis of IC and further investigations were carried out to assess their ability to carry out receptor-mediated endocytosis of soluble proteins/ molecules.

Ovalbumin $(\mathrm{OA})$ is generally regarded as being endocytosed through C-type lectin receptors [16] such as the macrophage mannose receptor CD206, which was strongly expressed by MoDCs (Fig. 6) [17] and fluorescent ovalbumin conjugates, Alexa-OA and DQ-OA, were used to assess the level of receptor-mediated capture and endocytosis of soluble molecules. DQ-OA is a self-quenched conjugate of ovalbumin that exhibits green fluorescence upon proteolytic degradation, which will occur after endocytosis. Cells representing immature MoDCs, blood $\mathrm{mDCs}$ and monocytes were incubated with equal amounts of Alexa-OA or DQ-OA and the capture of both Alexa-OA and DQ-OA were temperature dependent; shown by comparing experiments performed at 37 and at $4{ }^{\circ} \mathrm{C}$ (data not shown).

Upon proteolytic degradation, the fluorescence intensity of DQOA increases more than 10 times, which will, after total proteolysis, render one molecule of DQ-OA several times more fluorescent than one molecule of Alexa-OA. Both conjugates of OA should be recognized and captured at the molecular level by the same mechanism, however, capture and internalization of the conjugates into highly proteolytic endosomes will increase the fluorescence from the DQ-OA conjugate whereas the fluorescence from the Alexa conjugate will remain the same. Monocytes showed a quite higher and $\mathrm{mDCs}$ just a little bit higher fluorescence intensity after incubation with DQ-OA compared to Alexa-OA. MoDCs on the other hand showed an efficient capture of Alexa-OA, however, with a several magnitudes lower fluorescence intensity after incubation with DQ-OA (Fig. 7). MFI after incubation of three independent preparations of cells with Alexa-OA and DQ-OA are shown in Table 3. 
A Monocytes

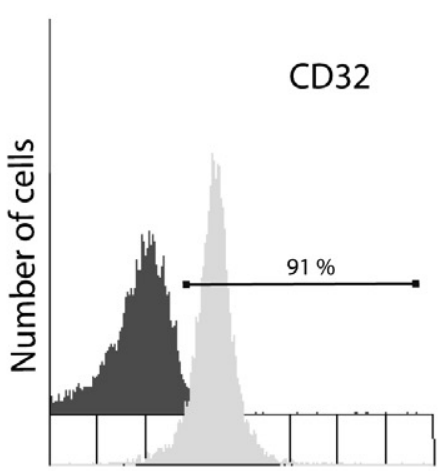

Fluorescence intensity

\section{B MoDCs}

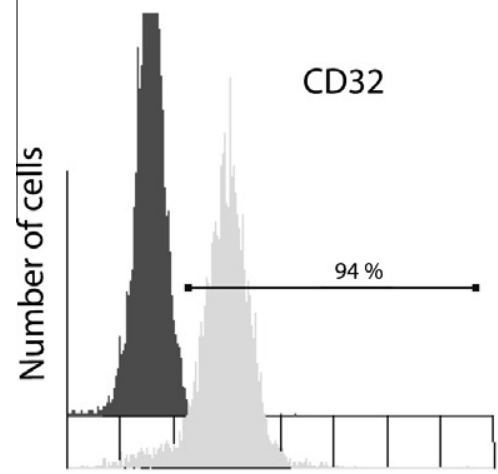

Fluorescence intensity

C Blood mDCs

CD32

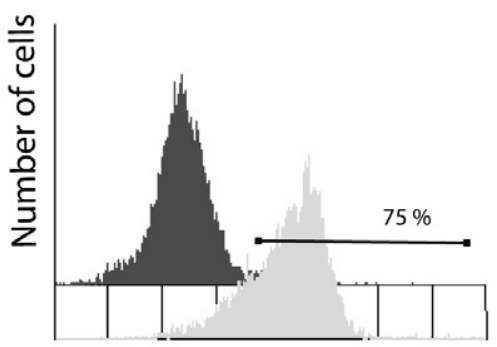

Fluorescence intensity

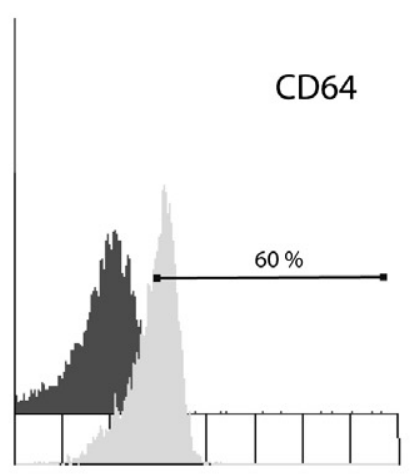

Fluorescence intensity

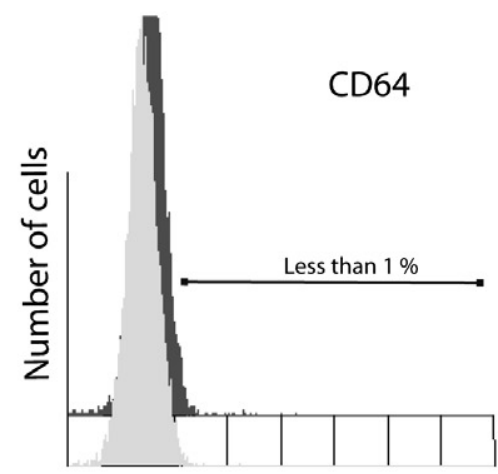

Fluorescence intensity

\section{CD64}

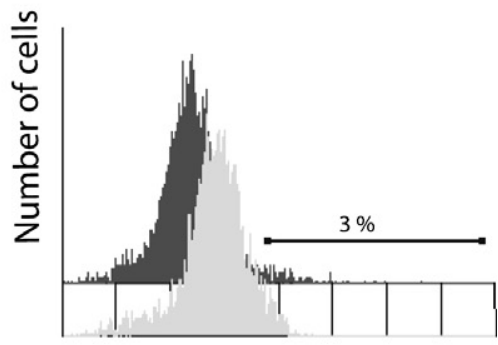

Fluorescence intensity

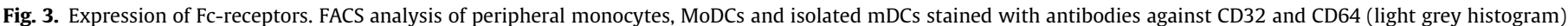

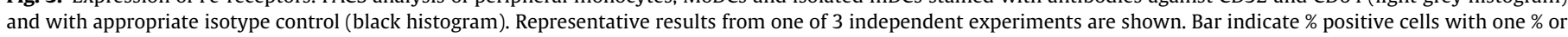
less positive cells in the isotype control.

Table 2

Expression of Fc-receptors CD32 and CD64 by human peripheral monocytes, MoDCs and blood mDCs. Mean fluorescence intensity (MFI) from three independent preparations of monocytes, MoDCs and blood mDCs after staining with antibodies against the Fc-receptors CD32 and CD64.

\begin{tabular}{lcll}
\hline Cell type & $\begin{array}{l}\text { Anti CD32 } \\
\text { MFI } \pm \text { sd }\end{array}$ & $\begin{array}{l}\text { Anti CD64 } \\
\text { MFI } \pm \text { sd }\end{array}$ & $\begin{array}{l}\text { Isotype control } \\
\text { MFI } \pm \text { sd }\end{array}$ \\
\hline Monocytes & $57 \pm 14$ & $28 \pm 3$ & $8 \pm 2$ \\
MoDCs & $29 \pm 9$ & $10 \pm 4$ & $5 \pm 2$ \\
mDC & $104 \pm 33$ & $15 \pm 11$ & $9 \pm 6$ \\
\hline
\end{tabular}

Capture of ovalbumin is generally regarded to be mediated by C-type lectin receptors and our results showed captured OA by monocytes and $\mathrm{mDCs}$ to be internalized in proteolytic endosomes whereas most of the OA captured by MoDCs was not proteolytically degraded.

\section{Discussion}

Human blood mDCs comprise only a small fraction of PBMC and a human blood mDC preparation contains several phenotypically and functionally different subpopulations $[12,18]$. Even though 
A MoDCs
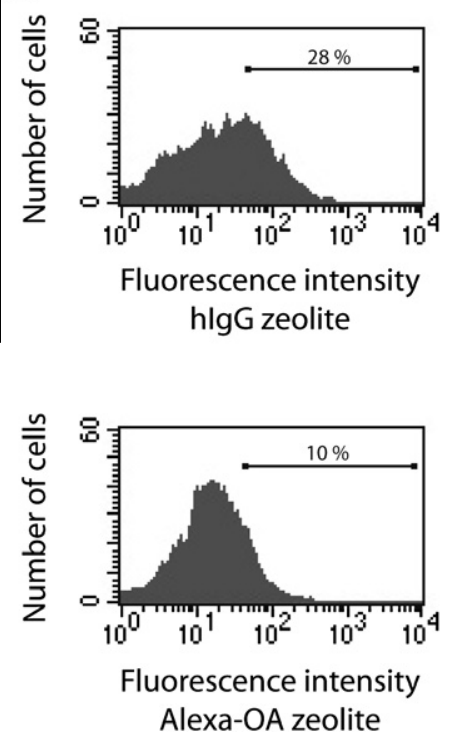

B Blood mDCs
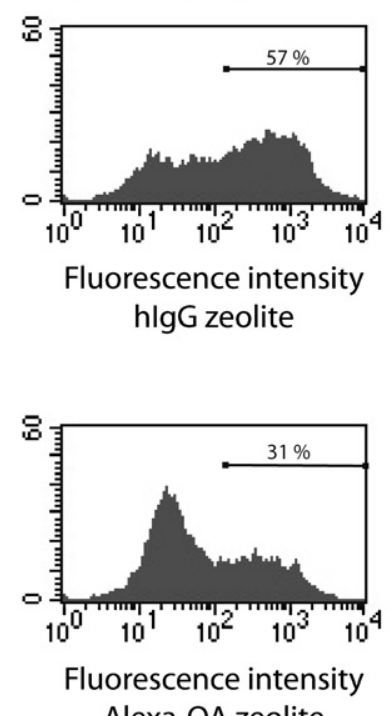

C Monocytes
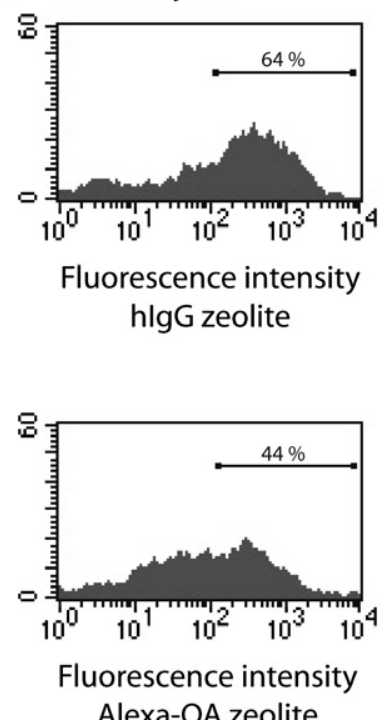

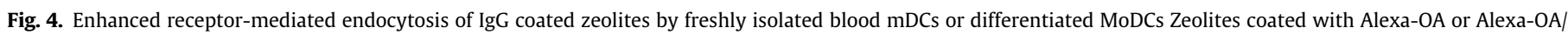

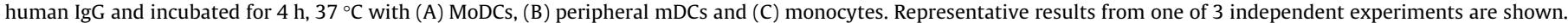

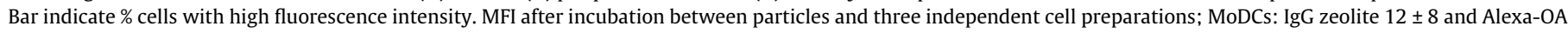
zeolite $9 \pm 5$, mDCs: IgG zeolite $186 \pm 43$ and Alexa-OA zeolite $100 \pm 49$ and monocytes: IgG zeolite $798 \pm 694$ and Alexa-OA zeolite $398 \pm 274$.

isolation of the $\mathrm{mDC}$ population generates cells expressing the surface marker $\mathrm{CD} 1 \mathrm{c}$, all of the cells might not be in the same development state or level of maturation and they may not share the same migratory capability. Human blood mDCs are in short supply and hence mDCs are generally generated from culture of peripheral CD14 ${ }^{+}$cells in a medium supplemented with IL- 4 and GM-CSF for 6-7 days [5]

As can be inferred from our results and also reported by others [12] the phenotype of the two DC subtypes shows similarities such as low expression of CD14, CD40, CD80 and high expression of CD11c and MHCII (Fig. 1). In our hands both peripheral mDCs and MoDCs differentiated from peripheral CD14 ${ }^{+}$cells represent an immature phenotype with very low expression of CD80 and 86 , both of which are regarded as maturation markers of DCs. However, culture and differentiation of MoDCs produce a more selected and homogeneous population of cells compared to isolated preparations of peripheral mDCs and these two DC populations are in several studies considered to be equal regarding antigen capture and processing.

Our data showed differences in the endocytic capacity towards zeolite particles (Fig. 2). Blood mDCs showed a high capacity to endocytose particles and more resembled monocytes in particle uptake than MoDCs. The capacity to bind and internalize particles can also be influenced by other elements such as surface charge and surface coating, e.g. opsoning molecules [19] In our experiments, co-adsorption of hIgG onto zeolite particles was used to facilitate receptor-enhanced endocytosis of the zeolite particles [15]. Although monocytes and both DCs populations clearly expressed Fc $\gamma$-receptors (Fig. 3), MoDCs were limited in the capture and endocytosis of hIgG-coated particles compared to blood mDCs and monocytes (Fig. 4).

According to earlier reports, the zeolite particles used had an average hydrodynamic diameter of $600 \mathrm{~nm}$ [20] and previous reports have shown that zeolite particles associated with cells after washing are almost completely localized intra-cellular with only a minor fraction adsorbed on the cell surface $[15,20,21]$. Consequently, particles firmly associated with cells after incubation and washing, are demonstrability phagocytosed by the cells in question. Protein aggregates such as IC and soluble proteins are smaller and less massive than zeolite particles and endocytosed through other intra cellular mechanisms [6]. Cellular association of IC and soluble molecules can be due to both adsorption on the external cell surface and endocytosed and this may explain the discrepancy observed regarding capture of IgG-coated zeolite particles and IC between MoDCs and blood mDCs (Figs. 4 and 5). As reported by others, uptake of IC is mainly mediated by Fc-receptors $[22,23]$, but association to the cell surface can also be mediated by non-internalizing, non-degradative Fc-receptors and other types of surface receptors such as the complement receptors [24-26]. In our hands, blood mDCs showed a low binding capacity with regard to IC; only a small number of the cells were able to capture IC, compared to MoDCs where the majority of the cells showed an efficient capture. In contrast to particle uptake, the efficient capture of IC by MoDCs and on the other hand a relatively low endocytosis of IgG coated zeolite particles, might be a reflection of a differential expression of receptors on the cell surface not detected by analysing cell surface CD32 and CD64. For instance are isoforms of CD32 ( $a$ and $b$ ) expressed by both MoDCs and mDCs mediating opposing effects on activation [27] and furthermore has Fc $\gamma$-receptors not internalized after binding IC, leaving captured IC on the surface, been identified $[15,24,25]$. Taken together our results indicate MoDCs, in comparison to blood mDCs, to express a relatively high portion of Fc $\gamma$-receptors not sustaining receptor mediated phagocytosis of large components as cells and cell debris.

Ovalbumin is commonly used to study mannose receptor-mediated endocytosis [28] as a model of uptake of soluble molecules and MoDCs showed after incubation with Alexa-OA a higher fluorescence intensity than the corresponding incubation with DQ-OA indicating a limited proteolysis and endocytosis of captured $\mathrm{OA}$ (Fig. 7). Both monocytes and blood mDCs showed a higher fluorescence intensity after incubation with DQ-OA compared to incubation with Alexa-OA indicating an efficient endocytosis of captured $\mathrm{OA}$, which distinguished the monocytes and the blood mDCs from the differentiated MoDCs. Our results suggest that a relatively large amount of captured ovalbumin and probably other glycoproteins captured by MoDCs will remain in a native state on the cell surface 


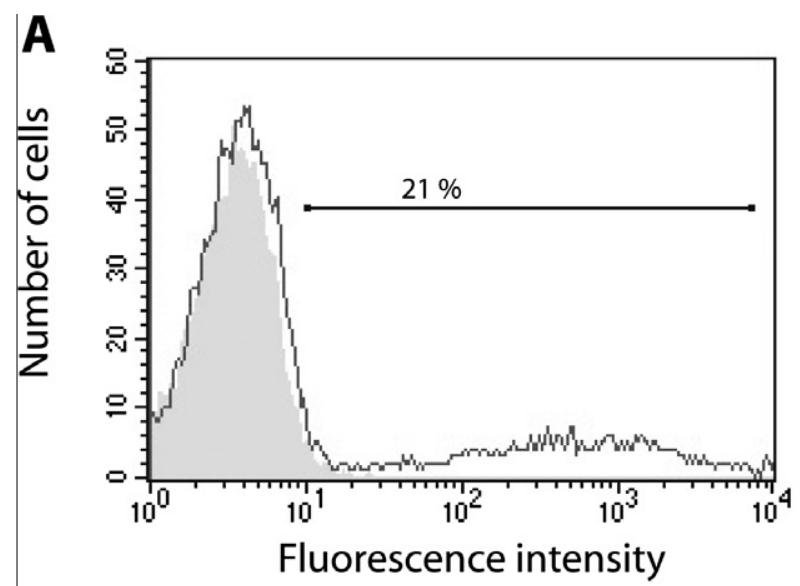

Blood mDCs

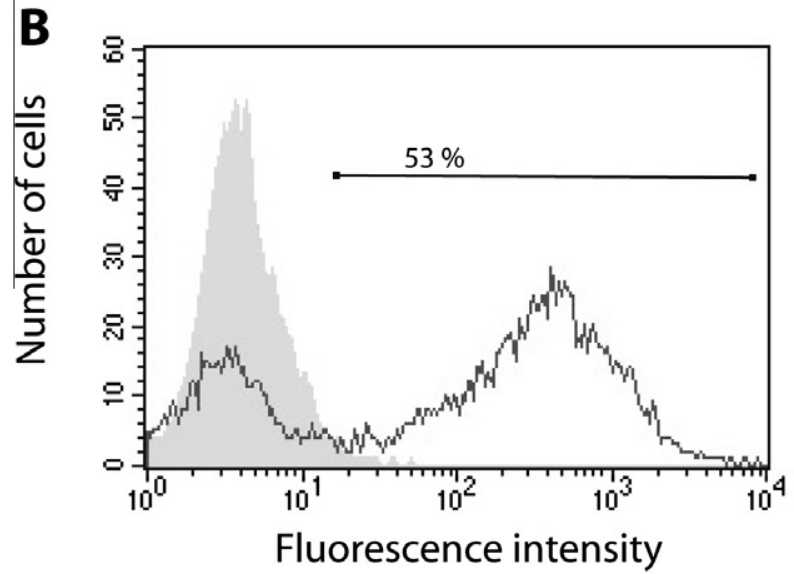

MoDCs

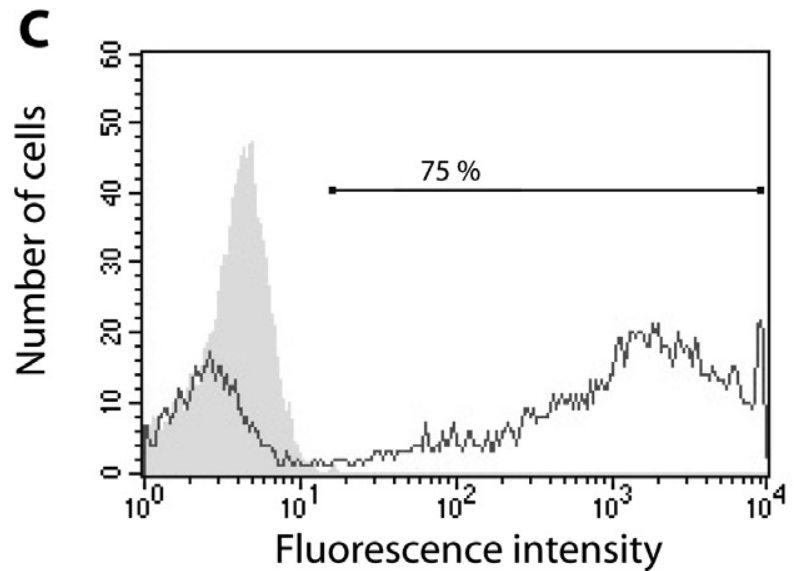

\section{Monocytes}

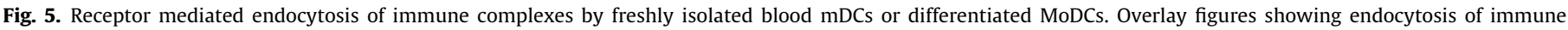

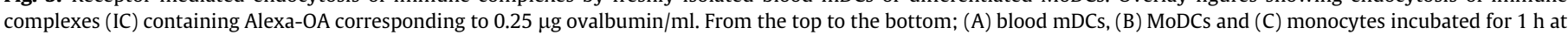

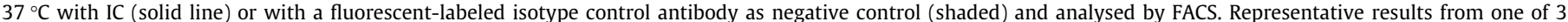

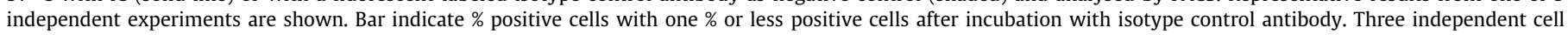

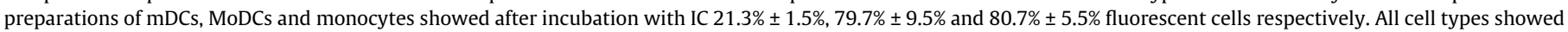
after incubation with control antibody $1.0 \% \pm 0.1 \%$ fluorescent cells.

and one explanation will be a high expression of non-internalizing lectin-like receptors in the MoDC population. C-type lectin receptors expressed by DCs are regarded to mediate capture of glycoproteins expressed by microorganisms or to mediate the association between DCs and lymphocytes [16]. A receptor that captures glycoproteins of microbial origin can be expected to mediate endocytosis into degrading endosomes while receptors mediating the association with other cells would be a non-internalizing receptor and captured ligands will thus not be transferred into proteolytic environments.

Monocytes used in this investigation were isolated by negative selection whereas peripheral mDCs were isolated by positive selection. No reports have shown any stimulating effects by anti BDCA-1 and MACS microbeads used in the isolation process of mDCs [29] and culture of isolated mDCs and differentiated MoDCs for $24 \mathrm{~h}$ in R10 medium showed no significant release of inflammatory 


\section{A. Monocytes}

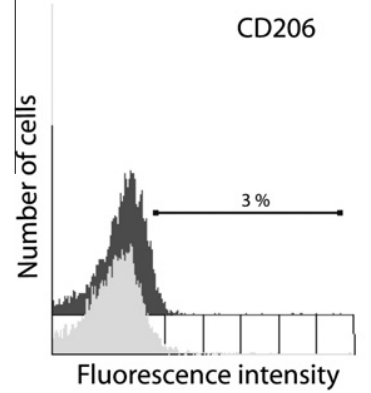

B. MoDCs

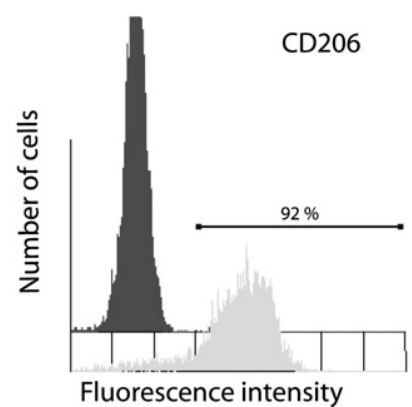

\section{Blood mDCs}

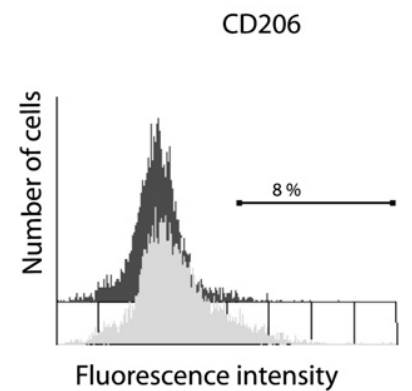

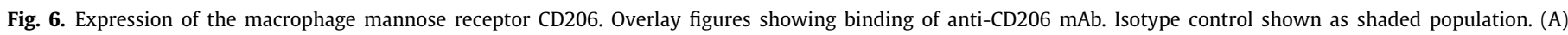

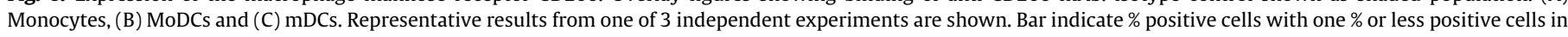

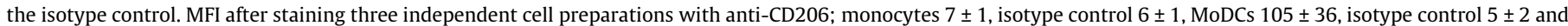
mDCs $15 \pm 11$, isotype control $8 \pm 7$.

\section{A. Monocytes}

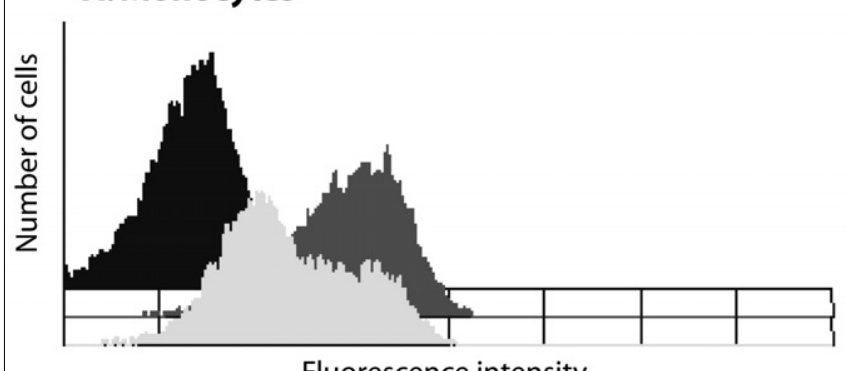

Fluorescence intensity

\section{B. Blood mDCs}

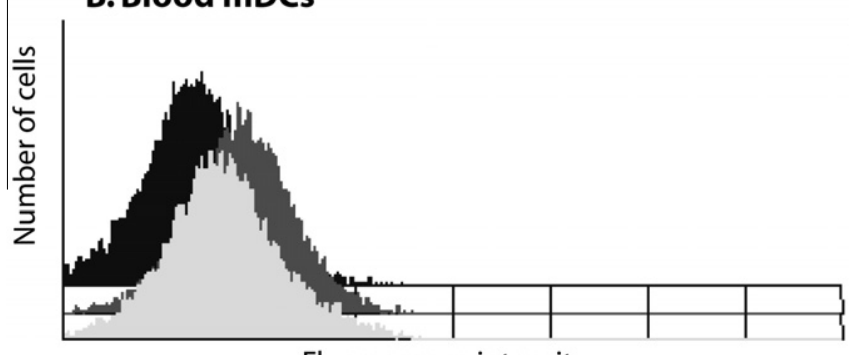

Fluorescence intensity

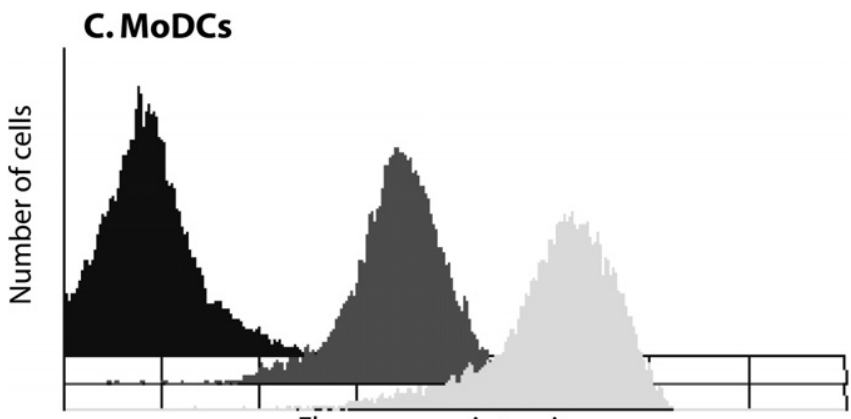

Fluorescence intensity

Fig. 7. Capture and endocytosis of ovalbumin. Overlay histograms showing fluorescence by (A) monocytes, (B) blood mDCs or (C) MoDCs after incubation with Alexa-OA or DQ-OA. Cells were incubated for $1 \mathrm{~h}$ at $37^{\circ} \mathrm{C}$ with $1 \mu \mathrm{g} / \mathrm{ml}$ Alexa$\mathrm{OA}$ or DQ-OA in the medium and analysed by FACS. Cells incubated with; Alexa-OA (light grey histogram), DQ-OA (dark grey histogram) or medium control (black histogram). Representative results from one of 3 independent experiments are shown.

cytokines into the culture medium. Cytokine concentrations in the collected medium were below or close to the detection limit when using multiplex assays as the Cytometric Bead Array System from
Table 3

Endocytosis of ovalbumin by human peripheral monocytes, blood mDCs and MoDCs. Mean fluorescence intensity (MFI) from three independent preparations of monocytes, MoDCs and mDCs after incubation with Alexa-OA or DQ-OA.

\begin{tabular}{lcll}
\hline Cell type & $\begin{array}{c}\text { Alexa-OA } \\
\text { MFI } \pm \text { sd }\end{array}$ & $\begin{array}{l}\text { DQ-OA } \\
\text { MFI } \pm \text { sd }\end{array}$ & $\begin{array}{l}\text { Un-stained } \\
\text { control MFI } \pm \text { sd }\end{array}$ \\
\hline Monocytes & $11 \pm 4$ & $31 \pm 3$ & $4 \pm 2$ \\
mDCs & $8 \pm 2$ & $12 \pm 4$ & $4 \pm 1$ \\
MoDC & $225 \pm 90$ & $27 \pm 18$ & $4 \pm 1$ \\
\hline
\end{tabular}

Becton Dickinson (data not shown). Although a reduction of the endocytosing capacity due to cross-linking of surface molecules on cells isolated by positive selection can't be completely ruled out. In our opinion cross-linking may reduce the phagocytosing capacity of isolated mDCs without effecting capture and endocytosis of IC and soluble glycoproteins as OA. Particles as zeolite particles are endocytosed by phagocytosis and the isolation process of our $\mathrm{mDC}$ preparation may under estimate the observed difference between MoDCs and mDCs regarding their capacity of capture and endocytose zeolite particles.

Peripheral monocytes, $\mathrm{CD} 14^{+}$cells, are differentiated in vitro into MoDCs or macrophages upon supplementation of the cultivation medium. In the presence of M-CFS, monocytes differentiate into macrophages whereas in the presence of GM-CSF and IL-4 the cells differentiate into MoDCs and to evaluate the generation of MoDCs, the phenotype of the cells are usually examined with antibodies against CD markers. Antibodies for phenotyping include antibodies recognizing CD14, maturation markers as CD80, CD83 or CD86, and C-type lectin receptors. Antibody "cocktails" with these antibody specifies are commercially available containing antibodies against CD14, CD83 and CD209, a C-type lectin receptor expressed by blood mDCs and diffentiated MoDCs [30]. CD209 however, is also expressed by macrophages and thus only discriminates MoDCs from monocytes, not from macrophages. Instead, using another antibody, against a C-type lectin receptor, the macrophage mannose receptor, CD206, not expressed by blood mDCs, confirmed the macrophage-like phenotype of our MoDC preparations (Fig. 6), which also has been reported earlier [31].

Several protocols describe the generation of DCs in vitro with respect to cell sources, cytokine concentrations, or culture period $[32,33]$. Although differentiated MoDCs and peripheral mDCs have shown expression of the same investigated surface markers, expression of surface markers by the generated DCs does not always reflect the functionality of the cells and other groups have reported that MoDCs and mDCs may differ in several ways, for example by their cytokine production in response to pathogen signals and ability to stimulate T lymphocytes [13,34]. There is a large 
interest in using DCs in clinical trails, in the fields of vaccination and cancer immunotherapy and most clinical studies have used in vitro generated MoDCs $[35,36]$. However, our results show that blood mDCs and MoDCs differ significantly in their performance of capture and endocytosis of particles, IC and soluble molecules. Interestingly, the MoDCs differed remarkably in the capture and processing of ovalbumin, which might be a reflection of the expression of surface receptors such as the C-lectin receptor CD206, or other types of C-lectin receptors [37].

Thus, based on our investigation, one can conclude a large difference in the endocytosing capacity and mechanisms between in vitro differentiated MoDCs and peripheral blood mDCs. Antigen uptake by APCs is usually mediated by several endocytosing mechanisms to achieve an efficient capture of foreign structures and molecules. However, care should be taken when uptake of isolated antigens, purified at a molecular level, are wanted by APCs since all APCs such as peripheral mDCs and differentiated MoDCs don't poses the same endocytosing capacity and mechanisms. MoDCs are valuable tools in DC biology, although it is of utmost importance to be aware of their endocytosing capabilities and mechanisms when MoDCs are used as APCs or as a model of antigen capture and presentation.

\section{References}

[1] Steinman RM, Inaba K, Turley S, Pierre P, Mellman I. Antigen capture processing, and presentation by dendritic cells: recent cell biological studies. Hum Immunol 1999;60:562-7.

[2] Mellman I, Steinman RM. Dendritic cells: specialized and regulated antigen processing machines. Cell 2001;106:255-8.

[3] Cao W, Lee SH, Lu J. CD83 is preformed inside monocytes, macrophages and dendritic cells, but it is only stably expressed on activated dendritic cells. Biochem J 2005;385:85-93.

[4] Banchereau J, Steinman RM. Dendritic cells and the control of immunity. Nature 1998;392:245-52.

[5] Sallusto F, Lanzavecchia A. Efficient presentation of soluble antigen by cultured human dendritic cells is maintained by granulocyte/macrophage colonystimulating factor plus interleukin 4 and downregulated by tumor necrosis factor alpha. J Exp Med 1994;179:1109-18.

[6] Mellman I. Endocytosis and molecular sorting. Annu Rev Cell Dev Biol 1996:12:575-625.

[7] Swanson JA, Watts C. Macropinocytosis. Trends Cell Biol 1995;5:424-8.

[8] Wilson NS, Villadangos JA. Regulation of antigen presentation and crosspresentation in the dendritic cell network: facts, hypothesis, and immunological implications. Adv Immunol 2005;86:241-305.

[9] Conner SD, Schmid SL. Regulated portals of entry into the cell. Nature 2006;422:37-44.

[10] Larsson K, Lindstedt M, Borrebaeck CA. Functional and transcriptional profiling of MUTZ-3, a myeloid cell line acting as a model for dendritic cells. Immunology 2006;117:156-66.

[11] Dahm A, Eriksson H. Ultra-stable zeolites-a tool for in-cell chemistry. J Biotechnol 2004;111:279-90.

[12] MacDonald KP, Munster DJ, Clark GJ, Dzionek A, Schmitz J, Hart DN. Characterization of human blood dendritic cell subsets. Blood 2002;100:4512-20.

[13] Osugi Y, Vuckovic S, Hart DN. Myeloid blood CD11c(+) dendritic cells and monocyte-derived dendritic cells differ in their ability to stimulate $T$ lymphocytes. Blood 2002;100:2858-66.

[14] Lindstedt M, Johansson-Lindbom B, Borrebaeck CA. Global reprogramming of dendritic cells in response to a concerted action of inflammatory mediators. Int Immunol 2002;14:1203-13.
[15] Hellman P, Andersson L, Eriksson H. Ligand surface density is important for efficient capture of immunoglobulin and phosphatidylcholine coated particles by human peripheral dendritic cells. Cell Immunol 2009;258:123-30.

[16] Figdor CG, van Kooyk Y, Adema GJ. C-Type lectin receptors on dendritic cells and Langerhans cells. Nat Rev Immunol 2002;2:77-84.

[17] Burgdorf S, Lukacs-Kornek V, Kurts C. The mannose receptor mediates uptake of soluble but not of cell-associated antigen for cross-presentation. J Immunol 2006;176:6770-6.

[18] Piccioli D, Tavarini S, Borgogni E, Steri V, Nuti S, Sammicheli C, et al. Functional specialization of human circulating CD16 and CD1c myeloid dendritic-cell subsets. Blood 2007;109:5371-9.

[19] Thiele L, Merkle HP, Walter E. Phagocytosis and phagosomal fate of surfacemodified microparticles in dendritic cells and macrophages. Pharm Res 2003;20:221-8.

[20] Andersson LI, Eriksson H. De-aluminated zeolite $\mathrm{Y}$ as a tool to study endocytosis, a delivery system revealing differences between human peripheral dendritic cells. Scand J Immunol 2007;66:52-61.

[21] Andersson LI, Hellman P, Eriksson H. Receptor-mediated endocytosis of particles by peripheral dendritic cells. Hum Immunol 2008;69:625-33.

[22] Amigorena S. Fc gamma receptors and cross-presentation in dendritic cells. J Exp Med 2002;195:F1-3.

[23] Larsson M, Berge J, Johansson AG, Forsum U. Human dendritic cells handling of binding, uptake and degradation of free and IgG-immune complexed dinitrophenylated human serum albumin in vitro. Immunology 1997;90:138-46.

[24] Bergtold A, Desai DD, Gavhane A, Clynes R. Cell surface recycling of internalized antigen permits dendritic cell priming of $B$ cells. Immunity 2005;23:503-14.

[25] Miettinen HM, Matter K, Hunziker W, Rose JK, Mellman I. Fc receptor endocytosis is controlled by a cytoplasmic domain determinant that actively prevents coated pit localization. J Cell Biol 1992;116:875-88.

[26] Kemper C, Atkinson JP. T-cell regulation: with complements from innate immunity. Nat Rev Immunol 2007;7:9-18.

[27] Boruchov AM, Veri M-C, Bonvini E, Ravetch JV, Young JW. Activating and inhibitory IgG Fc receptors on human DCs mediate opposing functions. J Clin Invest 2005;115:2914-23.

[28] Kindberg GM, Magnusson S, Berg T, Smedsrod B. Receptor-mediated endocytosis of ovalbumin by two carbohydrate-specific receptors in rat liver cells. The intracellular transport of ovalbumin to lysosomes is faster in liver endothelial cells than in parenchymal cells. Biochem J 1990;270:197-203.

[29] Dzionek A, Inagaki Y, Okawa K, Nagafune J, Röck J, Sohma Y, et al. Plasmacytoid dendritic cells: from specific surface marker to specific cellular functions. Hum Immunol 2002;63:1133-48.

[30] Teunis BH, Geijtenbeek TBH, Torensma R, Van Vliet SJ, Van Duijnhoven GCF, Adema GJ, et al. Identification of DC-SIGN, a novel dendritic cell-specific ICAM-3 receptor that supports primary immune responses. Cell 2000;100:575-85.

[31] Andreas Wollenberg A, Mommaas M, Oppel T, Schottdorf E-M, Günther S, Moderer M. Expression and function of the mannose receptor CD206 on epidermal dendritic cells in inflammatory skin diseases. J Invest Dermatol 2002;118:327-34.

[32] Conti L, Gessani S. GM-CSF in the generation of dendritic cells from human blood monocyte precursors: recent advances. Immunobiology 2008;213:859-70.

[33] Jacobs B, Wuttke M, Papewalis C, Seissler J, Schott M. Dendritic cell subtypes and in vitro generation of dendritic cells. Horm Metab Res 2008;40: 99-107.

[34] Jefford M, Schnurr M, Toy T, Masterman KA, Shin A, Beecroft T, et al. Functional comparison of DCs generated in vivo with Flt3 ligand or in vitro from blood monocytes: differential regulation of function by specific classes of physiologic stimuli. Blood 2003;102:1753-63.

[35] Banchereau J, Palucka AK. Dendritic cells as therapeutic vaccines against cancer. Nat Rev Immunol 2005;2005(5):296-306.

[36] Lesterhuis WJ, Aarntzen EH, De Vries IJ, Schuurhuis DH, Figdor CG, Adema GJ, et al. Dendritic cell vaccines in melanoma: from promise to proof? Crit Rev Oncol Hematol 2008;66:118-34.

[37] 't'Hart BA, van Kooyk Y. Yin-Yang regulation of autoimmunity by DCs. Trends Immunol 2004;25:353-9. 